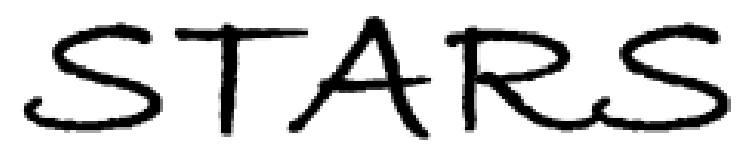

University of Central Florida

STARS

$1-1-2009$

\title{
Electrically induced deflective amplification for adaptive sensing of chemicals
}

\author{
Ruhai Tian \\ University of Central Florida \\ Liyuan Ma \\ University of Central Florida \\ Ming Su \\ University of Central Florida
}

Find similar works at: https://stars.library.ucf.edu/facultybib2000 University of Central Florida Libraries http://library.ucf.edu

This Article is brought to you for free and open access by the Faculty Bibliography at STARS. It has been accepted for inclusion in Faculty Bibliography 2000 s by an authorized administrator of STARS. For more information, please contactSTARS@ucf.edu.

\section{Recommended Citation}

Tian, Ruhai; Ma, Liyuan; and Su, Ming, "Electrically induced deflective amplification for adaptive sensing of chemicals" (2009). Faculty Bibliography 2000s. 2232.

https://stars.library.ucf.edu/facultybib2000/2232

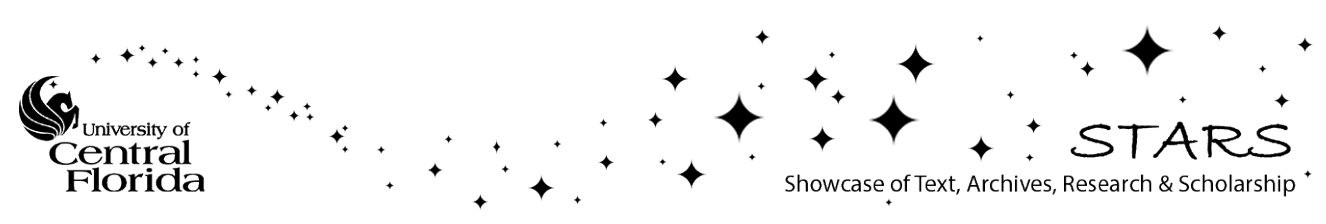




\section{Electrically induced deflective amplification for adaptive sensing of chemicals}

Cite as: Appl. Phys. Lett. 94, 013505 (2009); https://doi.org/10.1063/1.2999582

Submitted: 25 August 2008 . Accepted: 22 September 2008 . Published Online: 07 January 2009

Ruhai Tian, Liyuan Ma, and Ming Su

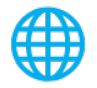

\section{Applied Physics Letters}

Mid-IR and THz frequency combs special collection 


\title{
Electrically induced deflective amplification for adaptive sensing of chemicals
}

\author{
Ruhai Tian, ${ }^{1,2}$ Liyuan Ma, ${ }^{1}$ and Ming Su ${ }^{1,2, a)}$ \\ ${ }^{1}$ NanoScience Technology Center, University of Central Florida, Orlando, Florida 32826, USA \\ ${ }^{2}$ Department of Mechanical, Materials and Aerospace Engineering, University of Central Florida, \\ Orlando, Florida 32826, USA
}

(Received 25 August 2008; accepted 22 September 2008; published online 7 January 2009)

\begin{abstract}
An adaptive strategy for sensitive detection of chemicals is proved in electrically induced deflections of an optical fiber. The surface of the fiber, close to one end, is modified by a sensing polymer film. Upon contact with solutions, the adsorption of ions onto the polymer changes the surface charge and bends the fiber in an electric field. The concept has been confirmed in several systems including detections of $p \mathrm{H}$ and metal ion, nanoparticles, and self-assembled monolayers. The dependence of the sensitivity on the strength of electric field provides an adaptive approach with adjustable sensitivity for chemical sensing. (C) 2009 American Institute of Physics.
\end{abstract}

[DOI: $10.1063 / 1.2999582$ ]

The highly sensitive detection of chemical using integrated devices is of great importance to many areas. ${ }^{1}$ Among available devices, a microcantilever sensor detects surface stresses of a thin solid beam induced by the preferential adsorption of molecules on one side of the microcantilever, which produces detectable mechanical deformations with nanoscale magnitude. ${ }^{2-5}$ The free energy change in the molecule-surface interaction, the geometry, and the Young's modulus of the cantilevers determine the sensitivity and operation range of these sensors. ${ }^{6}$ Once manufactured, such devices cannot detect target species with ultralow concentration (undetectable) or ultrahigh concentration (saturation) outside its operation range; in other words, the achievable sensitivity and the signal-to-noise ratio are intrinsically unadjustable. As a result, regardless of the chosen sensing system, the bending of a microcantilever at the end rarely exceeds $100 \mathrm{~nm}$. A variety of techniques including field effect transistor, optical level as well as chemical and mechanical amplification have been used to enhance detection sensitivity. ${ }^{7-9}$ In addition, when compared to ultrasensitive detection, the operation range has been ignored in the development of micro- and nanosensors. ${ }^{10-12}$

We propose the electrically induced deflective amplification (E-IDEA) for the adaptive, reconfigurable detection of chemicals using one-dimensional waveguide (optical fiber) for signal transduction (Fig. 1). The sensing materials (i.e., polymer) will coat the outer surface of the fiber close to the laser-emitting end. The surface charge changes upon the interactions with chemicals, which will lead to changes in the bending status of the fiber within an external electric field. Previously, the electric field induced motions have been studied in Faraday's demonstration of electrostatic repulsion between gold particles, in Millikan's oil droplet experiment to measure the unit charge of electron, and in electrophoresis to separate biomolecules. ${ }^{13,14}$ Most recently, the bending and vibration of carbon nanotubes and the motions of microparticles in electrostatic fields have been shown, ${ }^{15-18}$ but all of these are not in the scheme of adaptive chemical sensing.

The electric field induced mechanical deformations of optical fibers were measured on a home-built system. A

${ }^{a)}$ Electronic mail: mingsu@mail.ucf.edu. high voltage generator $(8000 \mathrm{~V})$ with a voltage fluctuation of less than $100 \mathrm{~V}$ was used to generate an electric field across the two parallel aluminum plates with surface areas of $1 \times 6 \mathrm{~cm}^{2}$. Changing the distance between the two electrodes adjusts the strength of the electric field. The electrode surfaces were insulated from the solution by coating a thin polymer layer or putting electrodes outside the solution. A collimated beam from a $3 \mathrm{~mW}$ helium neon laser $(633 \mathrm{~nm})$ was coupled into a multi mode optical fiber, which has a $190 \mu \mathrm{m}$ diameter silica core and a $30 \mu \mathrm{m}$ thick polymer shell with a uniform diameter and thickness over long distances. The superior mechanical property of the fiber offers a robust system with excellent repeatability. A position sensitive photodetector, which monitors the position of the emitted laser, was utilized to measure the fiber bending. The generated electric signals are preamplified and processed on a homemade electronic module and collected using an Agilent data acquisition unit $(11930 \mathrm{~A})$. To determine the displacement at the fiber end, the measured bending signal in voltage is multiplied by the sensitivity of the detector $(0.127 \mathrm{~V} / \mu \mathrm{m})$, which has been determined from the relationship between the voltage and the position change in the detector relative to the fiber end. The derived laser beam shift in the photodetector is then converted back to the displacement at the fiber end by considering the power of lens (2.3), the distance between the
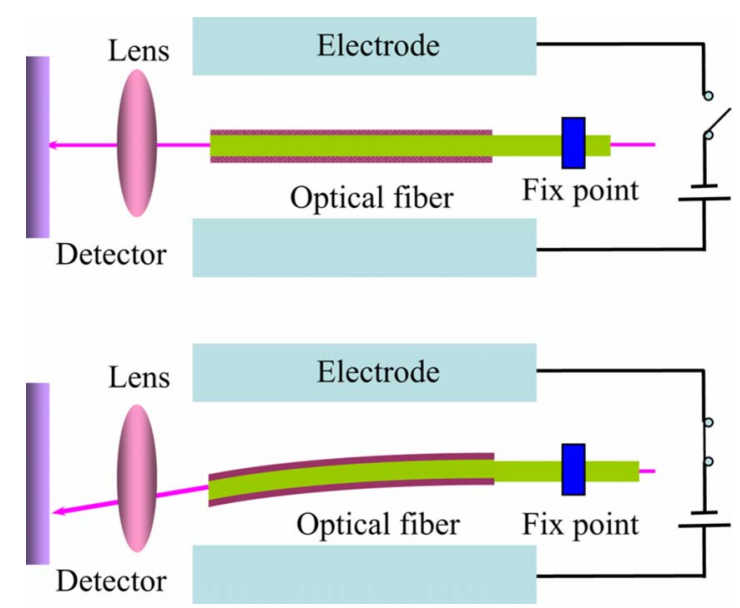

FIG. 1. (Color online) E-IDEA for adaptive molecular sensing. 

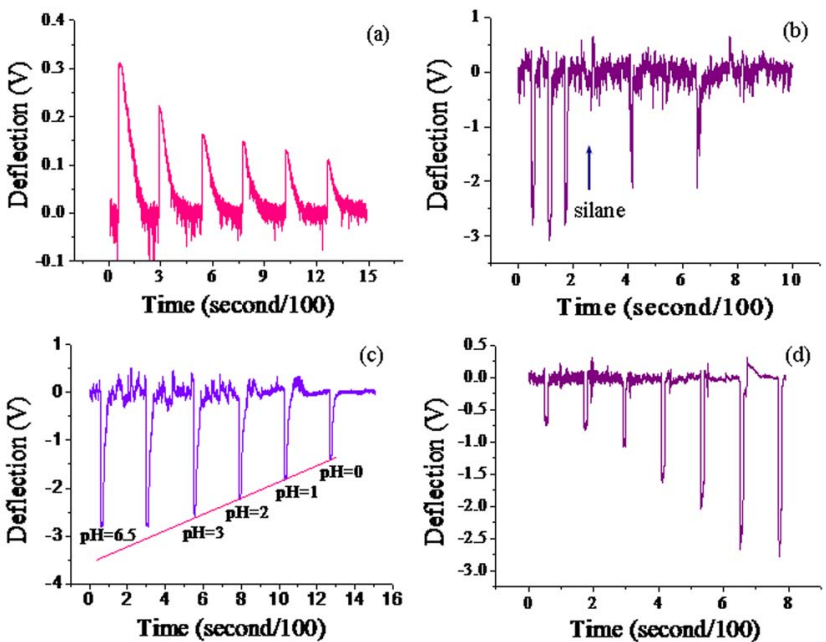

FIG. 2. (Color online) (a) The bending of a bare silica fiber in air with different electrode gaps increasing from $7 \mathrm{~mm}$ with a $1 \mathrm{~mm}$ step. The fiber length from the fixation point to the end is $7 \mathrm{~cm}$; (b) the bending of a bare fiber in a mixture of solvent upon addition of silane, the formation of a positively charged self-assembled monolayer will decrease the overall charge; (c) the bending of the polymer-coated fiber at different acidic $p \mathrm{H}$ values; and (d) the bending of a polymer-coated fiber in water with a $p \mathrm{H}$ of 6.5 with electrode gaps decreasing from $12 \mathrm{~mm}$ with a $1 \mathrm{~mm}$ step.

fiber end and the lens $(5 \mathrm{~cm})$, and the distance between the lens and the photodetector $(11.5 \mathrm{~cm})$. The pictures in Fig. 1 should be rotated $90^{\circ}$ with the detector sitting at the bottom in the real setup.

The polycarbonate coating on the optical fiber is removed by burning the polymer at one end. The silica core is then cleaned using concentrated sulfuric acid, which will negatively charge the surface with many hydroxyl groups. When an electric field is applied across the fiber, whether in air or in liquid, the fiber bends. The magnitude of bending depends on the dielectric constant of the media and the distance between the two electrodes. Figure 2(a) shows the bending of a bare silica fiber in air as a function of the electrode gap. The inverse relationship between the distance and the bending suggest that the bending is linearly dependent on the electric field strength, because the strength of the electric field inside the parallel electrodes depends inversely on the distance between two electrodes and the dielectric constant of the medium. The bending of the fiber in air, water, and ethanol is different due to the influence of the dielectric constants. The bending of a bare silica fiber during the formation of a self-assembled monolayer has been monitored. The chemically bonded monolayer will change the surface charge density of the silica surface. The fiber is first immersed in a solution that contains $50 \mathrm{ml}$ of methanol, $2 \mathrm{ml}$ of water, and $2 \mathrm{ml}$ of acetic acid. The bending of the fiber in an electric field is measured three times to ensure the system reaches the equilibrium state. After adding $2 \mathrm{ml}$ of 3 -aminopyltriethoxysilane to the solution, the bending of the fiber is measured by occasionally turning on the voltage and recording the fiber bending magnitudes. Figure 2(b) indicates that the magnitude of the fiber bending decreases where the other conditions are kept identical. This trend of change is in accordance to the decrease in the negative charge due to the formation of a position monolayer.

A $p \mathrm{H}$ sensitive polymer has been synthesized as follows: a solution containing $0.636 \mathrm{~g}$ of diaminoazobenzene, $0.654 \mathrm{~g}$ of pyromellitic dianhydride, and $14 \mathrm{ml}$ of dimethylacetamide was stirred for $20 \mathrm{~h}$ at $50-60{ }^{\circ} \mathrm{C}$. The mixture was poured in ethanol, yielding a viscous solution that can form stable and uniform thin films. After dipping a polycarbonate-coated fiber into the polymer solution for $1 \mathrm{~min}$, the polymer was soft baked at $60{ }^{\circ} \mathrm{C}$ to enhance the adhesion on the fiber. The thickness of the attached polymer layer is determined to be $10 \mu \mathrm{m}$ from its cross sectional optical micrograph. After immersion the modified end of the fiber into aqueous solutions with different $p \mathrm{H}$ values, hydrogen ions will preferentially adsorb onto the fiber and change the surface charge density and the bending status of the fiber in the electric field. Figure 2(c) shows the bending of the polymer-modified fiber in different $p \mathrm{H}$ solutions, which is measured by turning the voltage on for a short time, followed by turning the voltage off in order to change the $p \mathrm{H}$ of the solution. The purpose is to keep the effect of the electric field on the distributions of ions at a minimal level. The similar bending magnitude of the first two peaks in pure water confirms the repeatability of the experiment. When $p \mathrm{H}$ decreases from 4 to 0 , more hydrogen ions adsorb on the polymer, and the trend of decreasing magnitude of the fiber bending confirms the reduction in the surface charge. The bending magnitude decreases as the distance between the electrodes is decreased [Fig. 2(d)]. The scale of the bending roughly reflects the decrease in the strength of the electric field. Although the polymer responses well to acidic solution, it has poor adhesion in basic solution. The bending variation at small distance may be induced by the spatial variation in the electric field between electrodes with finite surface areas. In a control experiment using a bare silica fiber, the fiber bending cannot be reliably measured after dipping in different $p \mathrm{H}$ solutions. The reason is attributed to the complex interplay of charge adsorption on silica and surface dissolution.

The polycarbonate films around the optical fiber adsorb charged species from a solution and change the surface energy of the fiber. The polycarbonate is positively charged in neutral water. Upon the addition of negatively charged silica nanoparticles, the bending of the fiber in an electric field reverses in 3 min [Fig. 3(a)], which confirms the inversion of the overall surface charge from positive to negative. ${ }^{19}$ Considering the similarity between charged nanoparticles and proteins, this method can be extended to the detection of proteins. In addition, the adsorption of metal ions by polycarbonate has been used to detect silver ions contained in an aqueous solution. First, the polycarbonate layer on a fiber is swollen in a concentrated hydrogen chloride solution, increasing the pore size to encapsulate the chloride ions. After immersing the fiber with swollen polycarbonate in a silver nitride solution $\left(10^{-7} \mathrm{M}\right)$, the retained chloride ions can react with silver ions and produce a thin silver chloride layer on the fiber. Figure 3(b) shows the bending of the silver chloride coated fibers in air as a function of the immersion time, where the pink (1), purple (2), violet (3), and black (4) curves represent data collected at the immersion times of 0 , 1, 2 and $3 \mathrm{~min}$, respectively. A longer immersion time leads to a slower transition. Similarly, a higher concentration of silver nitride leads to a slower transition (not shown). The structure of the fiber also supports the results: silver chloride forms immediately on the outer surface of the swollen polymer, a longer immersion time or a higher silver ion concentration makes the silver chloride thicker. ${ }^{20}$ Chloride ions inside the shell diffuse throughout the weakly connected shell 

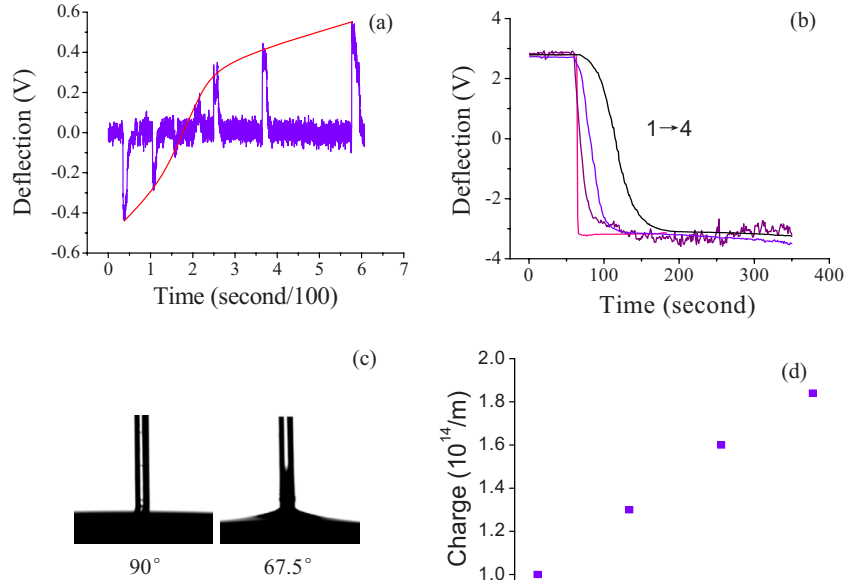

(c)

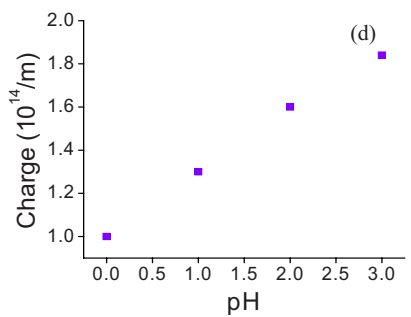

FIG. 3. (Color online) (a) The adsorption of a negatively charged silica nanoparticle on a polycarbonate-coated fiber in water will change the direction of the bending in an electric field. The fiber length is $7 \mathrm{~cm}$, and the gap distance is $9 \mathrm{~mm}$, (b) The bending of $\mathrm{HCl}$ treated polycarbonate-coated fiber in air after treatment with $10^{-7} \mathrm{M} \mathrm{Ag}^{+}$solution for 0 min (curve 1), $1 \mathrm{~min}$ (curve 2), 2 min (curve 3), and 3 min (curve 4), (c) The optical micrographs indicate the contact angles of a polycarbonate coated fiber and a fiber treated with concentrated hydrogen chloride, (d) The effective surface charge density on a polymer coated fiber derived from the data shown in Fig. 1(c).

in an electric field. The overall effective charge on the fiber changes as chloride ions diffuse out, owing to the different dielectric constants of silver nitride (11.2) and polycarbonate (3). The time dependent diffusion of ions is reflected in the difference in the transition time. The water contact angle measurements and atomic force micrograph (AFM) confirm the swollen of polycarbonate coating after immersions in solutions. Figure 3(c) indicates the contact angle of the same fiber with the nonswollen (left) and swollen (right) polycarbonate coatings, where the water contact angle decreases from $90^{\circ}$ to $67.5^{\circ}$, confirming that the surface coating becomes more hydrophilic after swelling. The contact mode AFM topographical images indicate a rougher surface after swelling (not shown). Energy dispersive x-ray analysis confirms the existence of silver: where the center silica core does not show silver, but the outer rim displays a strong silver signal (not shown).

We have derived the relationship between the bending, and the surface charge, diameter, and length of the fiber. The bending of fiber can be determined by equating the elastic force with the electrostatic force. Assuming a uniform charge distribution along the fiber, the electrostatic force on the fiber is uniform. The displacement at any point on the fiber is

$$
y(x)=-\frac{f x^{2}\left(6 L^{2}-4 x L+x^{2}\right)}{24 E I},
$$

where $x$ is the distance to the fixation point, $L$ is the length, $E$ is the Young's modulus, $f$ is the line load (electrostatic force per unit length) on the fiber, and $I$ is the moment of inertia of the fiber, that is, $I=\frac{1}{2} \mathrm{mr}^{2}$ for a solid cylinder of mass $m$ and radius $r$. The Young's modulus of the polymer coated silica fiber has been derived from $k(x)=3 \pi r^{4} / 4 x^{3} \cdot E$, where the spring constant $k$ is calculated by measuring the bending of horizontally suspended fibers with weights of known masses. Provided the electrodes spacing is $d$, the potential difference is $V$, and the effective charge density on fiber is $D$, the electrostatic force per unit length will be $f$ $=D V / d$. Since in the experiment, we have measured the maximal bending at the end of fiber that can be expressed as

$$
y_{\max }=y(L)=-\frac{f L^{4}}{8 E I} .
$$

Thus, the bending at any point can be related to the effect charge density on the fiber in

$$
y_{\max }=-\frac{D L^{4}}{8 E I} \frac{V}{d}=\frac{V}{4 E} \frac{L^{4}}{m r^{2} d} D .
$$

Thereby, the bending of the fiber depends on the electric field strength, the surface charge density, and mechanical property of the fiber. The effective charge density on a fiber is obtained from the bending at fiber end. Figure 3(d) shows the effective surface charge densities of a $7 \mathrm{~cm}$ long fiber in a $9 \mathrm{~mm}$ wide electrode gap, where the data are converted from the bending curve in Fig. 2(c) by considering Eq. (3), the sensitivity of the detection system, and the power of lens. The derived surface charge density is closed to that of metal oxide nanowire obtained using other methods. ${ }^{21}$ At last, this sensing mechanism is not limited to optical fiber but can be extended to microcantilever as well as other sensors. The universal applicability of the electric field induced deflection provides a general method for the adaptive chemical and biological sensing.

${ }^{1}$ M. Ferrari, Nat. Rev. Cancer 5, 161 (2005).

${ }^{2}$ P. J. Shaver, Rev. Sci. Instrum. 40, 901 (1969).

${ }^{3}$ G. Wu, H. Ji, K. Hansen, T. Thundat, R. Datar, R. Cote, M. F. Hagan, A. K. Chakraborty, and A. Majumdar, Proc. Natl. Acad. Sci. U.S.A. 98, 1560 (2001).

${ }^{4}$ J. Fritz, M. K. Baller, H. P. Lang, H. Rothuizen, P. Vettiger, E. Meyer, H. J. Guntherodt, C. Gerber, and J. K. Gimzewski, Science 288, 316 (2000).

${ }^{5}$ F. M. Battiston, J.-P. Ramseyer, H. P. Lang, M. K. Baller, Ch. Gerber, J. K. Gimzewski, E. Meyer, and H.-J. Guntherodt, Sens. Actuators B 77, 122 (2001).

${ }^{6}$ N. V. Lavrik, M. J. Sepaniak, and P. G. Datskos, Rev. Sci. Instrum. 75, 2229 (2004).

${ }^{7}$ G. Shekhawat, S. Tark, and V. P. Dravid, Science 311, 1592 (2006).

${ }^{8}$ M. Su, S.-Y. Li, and V. P. Dravid, Appl. Phys. Lett. 82, 3562 (2003).

${ }^{9}$ D. R. Evans, and V. S. J. Craig, J. Phys. Chem. B 110, 5450 (2006).

${ }^{10}$ M. Law, H. Kind, B. Messer, F. Kim, and P. Yang, Angew. Chem., Int. Ed. 41, 2405 (2005).

${ }^{11}$ S. Chopra, K. McGuire, N. Gothard, A. M. Rao, and A. Pham, Appl. Phys. Lett. 83, 2280 (2003).

${ }^{12}$ G. Zheng, F. Patolsky, Y. Cui, W. U. Wang, and C. M. Lieber, Nat Biotechnol. 23, 1294 (2005).

${ }^{13}$ R. A. Millikan, Nobel Lecture, 54 (1924).

${ }^{14}$ J. L. Viovy, Rev. Mod. Phys. 72, 813 (2000).

${ }^{15}$ P. Poncharal, Z. L. Wang, D. Ugarte, and W. A. De Heer, Science 283, 1513 (1999).

${ }^{16}$ T. Adrega, V. Chu, and J. P. Conde, Appl. Phys. Lett. 89, 143109 (2006).

${ }^{17}$ K.-I. Wada, K. Sasaki, and H. Masuhara, Appl. Phys. Lett. 81, 1768 (2002)

${ }^{18}$ S. T. Purcell, P. Vincent, C. Journet, and V. T. Binh, Phys. Rev. Lett. 89, 276103 (2002).

${ }^{19}$ J. Shiue, M. J. Matthewson, P. R. Stupak, and V. V. Rondinella, Acta Mater. 54, 2631 (2006).

${ }^{20}$ J. L. Armstrong, M. J. Matthewson, M. G. Juarez, and C. Y. Chou, Proc. SPIE 3848, 62 (1999).

${ }^{21}$ K.-I. Seo, S. Sharma, A. A. Yasseri, D. R. Steward, and T. I. Kamins, Electrochem. Solid-State Lett. 9, G69 (2006). 